\title{
Prevalence of cilantro (Coriandrum sativum) disliking among different ethnocultural groups
}

\author{
Lilli Mauer ${ }^{1}$ and Ahmed El-Sohemy ${ }^{2^{*}}$
}

\begin{abstract}
Background: Cilantro, the leaf of the Coriandrum sativum plant, is an herb that is widely consumed globally and has purported health benefits ranging from antibacterial to anticancer activities. Some individuals report an extreme dislike for cilantro, and this may explain the different cilantro consumption habits between populations. However, the prevalence of cilantro dislike has not previously been reported in any population. The objective of this study was to determine the prevalence of cilantro dislike among different ethnocultural groups from a population of young adults living in Canada. Subjects $(n=1,639)$ between the ages of 20 and 29 years were participants of the Toronto Nutrigenomics and Health Study. Individuals rated their preference for cilantro on a 9-point scale from 'dislike extremely' to 'like extremely'. Subjects also had the option to select 'have not tried' or 'would not try'. Subjects who selected 1 to 4 were classified as disliking cilantro.

Results: The prevalence of dislike ranged from 3 to $21 \%$. The proportion of subjects classified as disliking cilantro was 21\% for East Asians, 17\% for Caucasians, 14\% for those of African descent, 7\% for South Asians, 4\% for Hispanics, and 3\% for Middle Eastern subjects.

Conclusions: These findings show that the prevalence of cilantro dislike differs widely between various ethnocultural groups.
\end{abstract}

Keywords: Cilantro, Coriander, Flavor perception, Food preference

\section{Background}

Cilantro is one of the most polarizing and divisive food ingredients known. It has been well documented that those who like or dislike the herb provide extremely different descriptions of its flavor [1-3]. Individuals who like cilantro may describe it as fresh, fragrant or citrusy, whereas those who dislike cilantro report that it tastes like soap, mold, dirt or bugs, among other descriptors $[2,3]$. Numerous websites and online communities have been created to voice pro- or anti-cilantro opinions. This segregation is not seen with many common foods, which is why cilantro is of great interest to sensory scientists [1-3]. Most flavors do exhibit some degree of polarity, though it is rarely as extreme as that observed with cilantro. It has been documented that hereditary factors, along with exposure, shape our food preferences [4-6]. With the rising concern of global obesity, it is prudent to

\footnotetext{
* Correspondence: a.el.sohemy@utoronto.ca

${ }^{2}$ Department of Nutritional Sciences, University of Toronto, Room 310, 150 College St, Toronto, ON M5S 3E2, Canada

Full list of author information is available at the end of the article
}

determine the factors that determine acceptance of healthy foods [7].

Numerous factors influence food preferences, such as socio-cultural factors and genetics. Familiarity with certain foods also influences preference, and can affect the likelihood of trying new foods. Flavor, however, is one of the most important factors influencing food selection [8-10]. Perception of bitter, sweet, salty, sour and umami taste is mediated by clusters of taste receptor cells on the tongue, palate, larynx, oropharynx, epiglottis and esophagus. These receptors are scattered across the epithelial surface, and are interspersed with one another. Contrary to what was previously believed, there does not exist a clear map of taste regions across the tongue [11]. The current understanding is that signals are transduced to numerous gustatory areas of the brain by the binding of tastants to specific taste receptors [12]. The cholinergic system is thought to primarily mediate gustatory signal transduction; however, glutamate signaling has also been shown to be involved in establishment of conditioned taste aversions [13]. The amygdala and insular cortex are two areas that have been 
identified as being involved in consolidation and storage of gustatory memories, though research into the full extent of regions involved in this signaling is ongoing $[14,15]$. Formation of long-term memory involves new protein synthesis, whereas more rapid formation of short-term memory has been shown to be protein-independent. Formation of human taste memories is thus thought to involve novel protein synthesis; however, the time parameters of these processes are yet to be determined [16]. These memories are retrieved for comparison when a food is consumed, thus biological familiarity plays a role in taste preference. Similarly, olfaction is mediated by olfactory receptor neurons, which utilize a G-protein mechanism to transmit information about an odorant through the olfactory bulb to the olfactory cortex [17]. Odorant recognition helps the gustatory regions of the brain to identify the stimulus present in the oral cavity [12]. Perception of texture and consistency of foods is not mediated by one specific pathway, but could influence flavor intensity, likely by influencing the perception of tastants [18].

It is currently unknown whether strong reactions to the flavor of cilantro are a result of odorants or tastants. Whereas some research has investigated odorants [19], taste mechanisms have yet to be examined, although anecdotal evidence indicates that those who find cilantro offensive dislike the taste as well as the smell. Gas chromatography-olfactometry and CharmAnalysis have been used to identify the character-impact odorants in the oil of cilantro leaves [19]. Thirty-eight odor-active peaks were isolated from a sample of cilantro oil. Of those, the two trained panelists qualitatively described 33 eluted compounds in the same way, but two co-eluting odorant clusters were described differently by the two participants in the study [19]. Although only two individuals were involved in that study, the findings show that the odor profile is complex and perceived differently between individuals. Genetic factors are known to influence perception of certain odors and tastes [20], and twin studies have suggested strong heritability for cilantro preference $[1,3]$. However, no genetic factors associated with cilantro preference have yet been identified.

Anecdotally, the polarizing nature of cilantro has been well documented [1,3]; however, the prevalence of cilantro dislike remains unknown. This observational study aimed to determine the prevalence of cilantro dislike in different ethnocultural groups from a population of young adults.

\section{Results}

Table 1 shows the characteristics of the 1,381 subjects (419 male and 962 female) for which complete data were collected on all variables of interest. A total of $43 \%$ of females were Caucasian, which was significantly higher than the $40 \%$ of males who were Caucasian ( $P<0.0001$ ). A total of $41 \%$ of females were East Asian, which was
Table 1 Subject characteristics ${ }^{\mathrm{a}}$

\begin{tabular}{lcr}
\hline Characteristic & $\begin{array}{c}\text { Males } \\
(\mathbf{n}=\mathbf{4 1 9 )}\end{array}$ & $\begin{array}{r}\text { Females } \\
(\mathbf{n}=\mathbf{9 6 2})\end{array}$ \\
\hline Age, years & $22.9 \pm 2.5$ & $22.6 \pm 2.4$ \\
Ethnicity & $169(40)$ & $412(43)$ \\
Caucasian & $148(35)$ & $392(41)$ \\
East Asian & $63(15)$ & $102(11)$ \\
South Asian & $17(4)$ & $19(2)$ \\
Middle Eastern & $11(3)$ & $16(2)$ \\
Hispanic & $11(3)$ & $21(2)$ \\
African descent &
\end{tabular}

${ }^{a}$ Values are mean \pm standard deviation (SD) for continuous variables, and number (\%) for categorical variables.

significantly higher than the $35 \%$ of males who were East Asian $(\mathrm{P}<0.0001)$. Fifteen percent of males were South Asian compared to $11 \%$ of females who were South Asian $(P=0.002)$. No other ethnocultural groups had significantly different proportions of men and women.

Distribution of cilantro preference ratings in the population is shown in Table 2. The proportions of dislikers were not significantly different between men and women $(\mathrm{P}=0.15)$, with $14 \%$ of females and $10 \%$ of males being dislikers. No significant differences in the proportions of dislikers were observed between men and women in any ethnocultural group. However, the overall response distributions differed significantly between men and women when examining either the Caucasian or East Asian groups individually $(\mathrm{P}=0.02, \mathrm{P}=0.01)$. This was not the case with any other group, or in the population as a whole. The response distributions differed significantly between the ethnocultural groups $(\mathrm{P}<0.0001)$ with the Middle Eastern, Hispanic, and South Asian groups having the lowest proportions of dislikers (3\%, $4 \%$ and $7 \%$, respectively). The Hispanic and South Asian groups both also had significantly higher proportions of likers than any other groups (92\% and $75 \%$, respectively; $\mathrm{P}<0.001$ ). A high proportion of East Asians, Caucasians and individuals of African descent had never tried cilantro (27\%, $16 \%$ and $31 \%$, respectively); these groups also had the highest prevalence of dislikers. The proportion of individuals who would not try cilantro was highest among East Asians at 1.1\%.

Figure 1 shows the distribution of cilantro preference ratings on the 9-point scale for the three major ethnocultural groups: Caucasians, East Asians and South Asians. This histogram shows the specific breakdowns (the numeric responses selected) of liker, neutral and disliker categories.

Table 3 shows the distribution of leaf lettuce preferences among the ethnocultural groups. This demonstrates a typical preference distribution for a food that is considered non-polarizing. The most frequently selected preference response for this food was 7 (like moderately) within each 
Table 2 Cilantro preference distributions between different ethnocultural groups

\begin{tabular}{|c|c|c|c|c|c|}
\hline \multicolumn{6}{|c|}{ Preference category $^{a}$} \\
\hline & \multicolumn{3}{|c|}{ Have tried $^{\mathbf{b}}$} & \multicolumn{2}{|c|}{ Have not tried ${ }^{c}$} \\
\hline & Like & Neutral & Dislike & Never tried & Would not try \\
\hline Caucasian $(n=581)$ & $311(64)$ & $88(18)$ & $85(17)$ & $96(16)$ & $1(0.2)$ \\
\hline East Asian $(n=540)$ & $207(53)$ & $102(26)$ & $81(21)$ & $144(27)$ & $6(1.1)$ \\
\hline South Asian $(n=165)$ & $119(75)$ & $27(17)$ & $11(7)$ & $8(5)$ & 0 \\
\hline Middle Eastern $(n=36)$ & $8(69)$ & $20(28)$ & $1(3)$ & $7(19)$ & 0 \\
\hline Hispanic $(n=27)$ & $24(92)$ & $1(4)$ & $1(4)$ & $1(4)$ & 0 \\
\hline African descent $(n=32)$ & $13(59)$ & $6(27)$ & $3(14)$ & $10(31)$ & 0 \\
\hline
\end{tabular}

${ }^{\text {a }}$ Subjects selecting 1 to 4 are classified as dislikers, 5 are neutral, 6 to 9 are likers;

b Values are $\mathrm{n}(\%)$ of subjects who have tried cilantro.

c Values are $n(\%)$ of ethnocultural group.

ethnocultural group. The prevalence of dislikers ranged from 0 to $6 \%$. A significantly lower proportion of individuals within each ethnocultural group reported disliking leaf lettuce as compared to cilantro (Caucasian: $\mathrm{P}<0.0001$, East Asian: $\mathrm{P}<0.0001$, South Asian: $\mathrm{P}=0.02$ ).

\section{Discussion}

Despite the well-recognized extreme differences in cilantro preference between individuals [1], no study has previously reported the prevalence of this trait in any population. In the present study, we examined the prevalence of cilantro dislike in different ethnocultural groups from a convenience sample of young Canadian adults recruited from the University of Toronto campus. We observed a difference in the distribution of preferences between the different ethnocultural groups as well as between men and women among certain ethnocultural groups, which may be attributed to both biological and social factors.

The Middle Eastern, Hispanic and South Asian groups had the lowest proportions of cilantro dislikers. This may be due to frequency of exposure, as cilantro is most popular in these styles of cuisine [21], and culture does modify

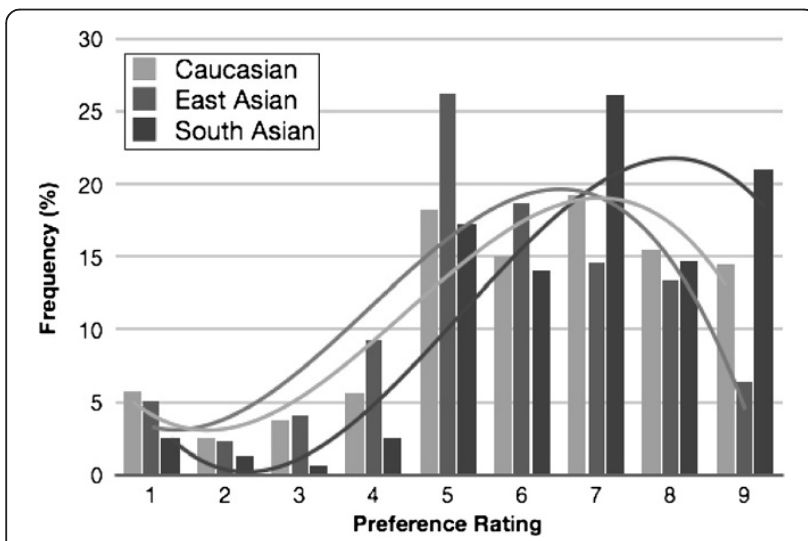

Figure 1 Cilantro preference distribution histogram for Caucasian, East Asian, and South Asian subjects. food-related behaviors [22]. The lower prevalence of cilantro dislike among these groups could also be due to genetic differences influencing cilantro flavor perception. East Asians and Caucasians had the highest prevalence of cilantro dislikers. One limitation of our study was that the East Asian group included individuals of Thai, Korean, Japanese, Vietnamese and Chinese descent. Cilantro may be more widely used in certain East Asian cuisines, such as Thai and Vietnamese [23], and less so in others, which may have influenced our estimated proportions of East Asians who dislike or have never tried cilantro $(21 \%$ and $27 \%$, respectively). Furthermore, the Caucasian group also consisted of individuals from a wide variety of European countries. Dietary patterns vary greatly between the different regions of Europe and it was not possible to distinguish whether regional differences may have influenced cilantro preference responses in our large, heterogeneous Caucasian group. It should also be noted that the numbers of subjects within some of the ethnocultural groups was much smaller than for Caucasians and East Asians. Nonetheless, differences were observed between ethnicities. It has been suggested that genetic factors may be responsible for differential perception of the flavor of cilantro [1]. Genetic heterogeneity between ethnocultural groups may thus contribute to the different preference distributions.

Table 3 shows the preference distribution of leaf lettuce, an example of a common food that is considered to be non-polarizing. Among each ethnocultural group, the response distribution curves were normal, with peaks at 7 (like moderately). Similar findings would be expected when examining most common foods. While leaf lettuce likers and dislikers seem to exist, reactions are not extreme. This underscores the unusual, divisive nature of cilantro.

Because qualitative descriptions of the flavor of cilantro differ considerably between those who like and dislike it, differences in perception of the flavor are likely driving the observed differences in preference. Whether this is due to differential perception of an odorant or tastant, or both, is currently unknown $[1,3]$. It may be that individuals who 
Table 3 Leaf lettuce preference distributions between different ethnocultural groups

\begin{tabular}{|c|c|c|c|c|c|}
\hline \multicolumn{6}{|c|}{ Preference category $^{a}$} \\
\hline & \multicolumn{3}{|c|}{ Have tried $^{\mathrm{b}}$} & \multicolumn{2}{|c|}{ Have not tried ${ }^{c}$} \\
\hline & Like & Neutral & Dislike & Never tried & Would not try \\
\hline Caucasian $(n=581)$ & $518(89)$ & $50(9)$ & $11(2)$ & $2(0.3)$ & 0 \\
\hline East Asian $(n=540)$ & $441(82)$ & $78(15)$ & $17(3)$ & $4(0.7)$ & 0 \\
\hline South Asian $(n=165)$ & $133(84)$ & $22(14)$ & $3(2)$ & $7(4.2)$ & 0 \\
\hline Middle Eastern $(n=36)$ & $36(100)$ & 0 & 0 & 0 & 0 \\
\hline Hispanic $(n=27)$ & $26(96)$ & 0 & $1(4)$ & 0 & 0 \\
\hline African Descent $(n=32)$ & $23(74)$ & $6(19)$ & $2(6)$ & $1(3)$ & 0 \\
\hline
\end{tabular}

a Subjects selecting 1 to 4 are classified as dislikers, 5 are neutral, 6 to 9 are likers;

${ }^{b}$ Values are $\mathrm{n}(\%)$ of subjects who have tried leaf lettuce.

c Values are $n(\%)$ of ethnocultural group.

dislike cilantro are anosmic to one or more of the pleasant smelling compounds found in cilantro. Alternatively, those who like cilantro may be anosmic to an unpleasant smelling compound - perhaps an aldehyde that, alone, smells of soap [3]. E-(2)-Decenal has been proposed as a candidate compound, as it is emitted by stink bugs and other insects in defensive secretions $[24,25]$. It has been suggested that this may be one of the compounds in cilantro that individuals find unpleasant, although this has not yet been tested. Because of the complex chemical composition of the oil of cilantro leaves, there are many potential candidates. Considering there are approximately 350 olfactory receptor genes and another 300 or more olfactory receptor pseudogenes of unknown function $[17,26]$, there are many potential candidates that could explain inter-individual differences in cilantro preference. The interaction between taste and olfaction is well-established [27], but it remains unclear whether one is more influential than the other with respect to cilantro preference.

Although differences in flavor perception, possibly attributable to genetic differences between ethnocultural groups, is likely responsible for the different distributions of cilantro preference seen, we cannot rule out the possibility of differences in exposure and use of cilantro in the traditional cuisines of different ethnocultural groups driving differences in preference. The relationship between flavor perception, familiarity and exposure, and preference is complex and cannot be further explored in the present study. Future studies will need to be conducted to determine the cause of the different preference distributions observed in this study.

It should be noted that the current study consists of a convenience sample of young adults recruited from the University of Toronto campus and results may not reflect older adults or the Canadian population in general. Further studies will be required to assess the prevalence of cilantro liking and disliking in the broader population and among other ethnocultural groups.

\section{Conclusions}

In summary, we report that cilantro dislike varies from $3 \%$ to $21 \%$ in this population of young adults depending on the ethnocultural group. The contribution of individual genetic differences to this trait remains to be determined.

\section{Methods}

\section{Subjects}

Participants ( $\mathrm{n}=1,639 ; 1,117$ women and 522 men) were enrolled in the Toronto Nutrigenomics and Health Study, which is a cross-sectional study investigating gene-diet interactions and biomarkers of chronic disease, as well as genetic determinants of eating behaviors. Subjects between 20 and 29 years of age were recruited from the University of Toronto campus. Subjects were excluded if they were pregnant or breastfeeding, due to metabolic and dietary changes that take place during this period. Subjects who could not communicate in English, or who did not provide a 12-hour fasting venous blood sample were also excluded. Smokers $(n=105)$ were excluded from the present analysis because of the known effects of smoking on taste and odor perception [17]. Subjects with any missing data were also excluded $(n=10)$. At the time of screening, subjects identified the ethnocultural group(s) they belong to. Subjects who listed more than one ethnicity $(n=143)$ or any group with fewer than 20 subjects were excluded from the current analyses, and the remaining individuals were classified into one of six groups (Caucasian, $\mathrm{n}=581$; East Asian, $\mathrm{n}=540$; South Asian, $\mathrm{n}=165$; Middle Eastern, $\mathrm{n}=36$; African descent, $n=32$; and Hispanic, $n=27$ ). After exclusions, the final sample population consisted of 1,381 subjects (962 women and 419 men). All subjects provided written informed consent, and the University of Toronto Research Ethics Board approved the study protocol.

\section{Cilantro preference data collection}

Subjects completed a 63-item food preference checklist, which included a range of common foods and beverages, 
as well as food garnishes and condiments. Participants gave each item a rating from 1 (dislike extremely) to 9 (like extremely). Alternatively, subjects had the option of selecting 'never tried' or 'would not try'.

\section{Statistical analysis}

All statistical analyses were conducted using Statistical Analysis Systems software (SAS version 9.2; SAS Institute, Cary, NC, USA). The frequency procedure was used to compare preference responses between ethnocultural groups, and $\chi^{2}$ tests were used to examine differences between preference distributions. Dislikers were defined as those reporting 1, 2, 3 or 4 (dislike extremely, dislike very much, dislike moderately, dislike slightly) on the 9-point scale. Those selecting 5 (neither like nor dislike) were classified as neutral, and those selecting 6, 7, 8 or 9 (like slightly, like moderately, like very much, like extremely) were classified as likers. The mean and median ratings fell to the right of the arithmetic center of the scale (6.08 and 6 , respectively), suggesting a slightly skewed distribution, which was confirmed using a Shapiro-Wilk test for normality. Those selecting 'never tried' were included in the analyses in order to examine the ethnocultural breakdown of this group. Those selecting 'would not try' were also included in the analyses since some of these individuals may dislike the odor so strongly that they would never consume cilantro. For comparison, leaf lettuce preference distributions were examined using the same methods. Leaf lettuce is a food commonly used as a garnish, but is not known to elicit the same polarizing responses as cilantro.

\section{Competing interests}

The authors declare they have no competing interests.

\section{Authors' contributions}

LM completed statistical analyses and prepared the first draft of the manuscript. AE obtained funding and provided supervision. Both authors contributed to data interpretation and critical review of the manuscript for important intellectual content. Both authors read and approved the final manuscript.

\section{Authors' information}

AE holds a Canada Research Chair in Nutrigenomics.

\section{Acknowledgements}

Grant funding was supplied by the Advanced Foods and Materials Network Centre of Excellence (AFMNet). The funding body had no role in the design, collection, analysis, interpretation, writing or publication of this manuscript.

\section{Author details \\ 'Department of Nutritional Sciences, University of Toronto, Toronto, Canada. ${ }^{2}$ Department of Nutritional Sciences, University of Toronto, Room 310, 150} College St, Toronto, ON M5S 3E2, Canada.

Received: 21 October 2011 Accepted: 20 January 2012

Published: 2 May 2012

\section{References}

1. Herz RS: I know what i like: understanding odor preferences, The Smell Culture Reader. Providence, Rl: Oxford: Berg; 2004:190-203.

2. McGee H: Cilantro haters, it's not your fault. New York City: The New York Times; 2010:1.
3. Rubenstein S: Across the land, people are fuming over an herb (no, not that one). New York City: The Wall Street Journal; 2009:1.

4. Capaldi EP: (Ed): Why We Eat What We Eat: the Psychology Of Eating. Washington: American Psychological Association; 1996.

5. Cowart BJ: Development of taste perception in humans: Sensitivity and preference throughout the life span. Psychol Bull 1981, 90:43-73.

6. Rozin P: Development in the food domain. Dev Psychol 1990, 26:555-562.

7. Eertmans A, Baeyens F, Van den Bergh O: Food likes and their relative importance in human eating behavior: review and preliminary suggestions for health promotion. Health Educ Res 2001, 16:443-456.

8. Fallon $A E$, Rozin P: The psychological bases of food rejections by humans. Ecol Food Nutr 1983, 13:15-26.

9. Birch LL: Development of food preferences. Ann Rev Nutr 1999, 19:41-62.

10. Drewnowski A: Taste preferences and food intake. Ann Rev Nutr 1997, 17:237-253.

11. Chandrashekar J, Hoon M, Ryba N, Zuker C: The receptors and cells for mammalian taste. Nature 2006, 444:288-294.

12. Kobayashi M: Functional organization of the human gustatory cortex. J Oral Biosci 2006, 48:244-260.

13. Bermúdez-Rattoni F, Ramírez-Lugo L, Gutiérrez R, Miranda MI: Molecular signals into the insular cortex and amygdala during aversive gustatory memory formation. Cell Mol Neurobiol 2004, 24:25-36.

14. Bermudez-Rattoni F, Nunez-Jaramillo L, Balderas I: Neurobiology of Taste-recognition Memory Formation. Chem Senses 2005, 30:i156-i157.

15. Behrens M, Meyerhof W: Gustatory and extragustatory functions of mammalian taste receptors. Physiol Behav 2011, 105:4-13.

16. Houpt TA, Berlin R: Rapid, labile, and protein synthesis - independent shortterm memory in conditioned taste aversion. Learn Mem 1999, 6:37-46.

17. Buck LB: Olfactory receptors and odor coding in mammals. Nutr Rev 2004, 62:184-188. discussion S224-141.

18. Hollowood TA, Linforth RST, Taylor AJ: The effect of viscosity on the perception of flavour. Chem Senses 2002, 27:583-591.

19. Eyres G, Dufour J-P, Hallifax G, Sotheeswaran S, Marriott PJ: Identification of character-impact odorants in coriander and wild coriander leaves using gas chromatography-olfactometry (GCO) and comprehensive twodimensional gas chromatography-time-of-flight mass spectrometry (GC × GC-TOFMS). J Sep Sci 2005, 28:1061-1074.

20. Reed DR, Knaapila A: Genetics of taste and smell poisons and pleasures. Prog Mol Biol Transl Sci 2010, 94:213-240.

21. Wong PYY, Kitts DD: Studies on the dual antioxidant and antibacterial properties of parsley (Petroselinum crispum) and cilantro (Coriandrum sativum) extracts. Food Chem 2006, 97:505-515.

22. Axelson ML: The impact of culture on food-related behavior. Ann Rev Nutr 1986, 6:345-363.

23. Cadwallader Keith R, Benitez D, Pojjanapimol S, Suriyaphan O, Singh T: Characteristic aroma components of the cilantro mimics, Natural Flavors and Fragrances. Washington, DC: American Chemical Society: Frey C, Rouseff R; 2005:117-128.

24. Potter TL: Essential oil composition of cilantro. J Agric Food Chem 1996, 44:1824-1826.

25. Borges M, Aldrich JR: Instar-specific defensive secretions of stink bugs (Heteroptera: Pentatomidae). Cell Mol Life Sci 1992, 48:893-896.

26. Malnic B, Godfrey PA, Buck LB: The human olfactory receptor gene family. Proc Natl Acad Sci U S A 2004, 101:2584-2589.

27. Murphy C, Cain WS: Taste and olfaction: independence vs interaction. Physiol Behav 1980, 24:601-605

doi:10.1186/2044-7248-1-8

Cite this article as: Mauer and El-Sohemy: Prevalence of cilantro

(Coriandrum sativum) disliking among different ethnocultural groups.

Flavour 2012 1:8 